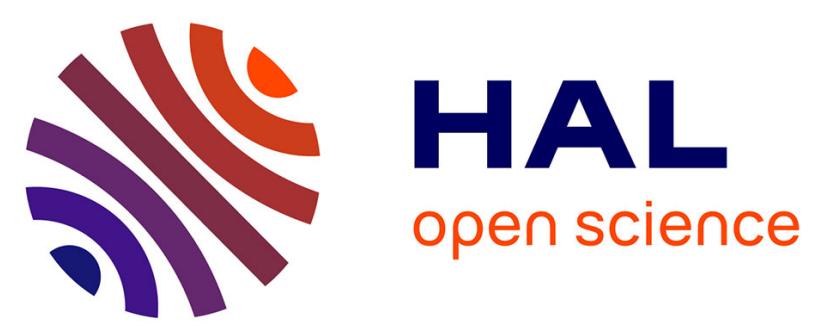

\title{
Guaranteed cost for an event-triggered consensus strategy for interconnected two time-scales systems with structured uncertainty
}

Yan Lei, Yan-Wu Wang, Irinel-Constantin Morarescu, Jiang-Wen Xiao

\section{- To cite this version:}

Yan Lei, Yan-Wu Wang, Irinel-Constantin Morarescu, Jiang-Wen Xiao. Guaranteed cost for an eventtriggered consensus strategy for interconnected two time-scales systems with structured uncertainty. IEEE Transactions on Cybernetics, In press, pp.Early Access. 10.1109/TCYB.2020.3026352 . hal02956229

\section{HAL Id: hal-02956229 \\ https://hal.science/hal-02956229}

Submitted on 2 Oct 2020

HAL is a multi-disciplinary open access archive for the deposit and dissemination of scientific research documents, whether they are published or not. The documents may come from teaching and research institutions in France or abroad, or from public or private research centers.
L'archive ouverte pluridisciplinaire HAL, est destinée au dépôt et à la diffusion de documents scientifiques de niveau recherche, publiés ou non, émanant des établissements d'enseignement et de recherche français ou étrangers, des laboratoires publics ou privés. 


\title{
Guaranteed cost for an event-triggered consensus strategy for interconnected two time-scales systems with structured uncertainty
}

\author{
Yan Lei, Yan-Wu Wang, Irinel-Constantin Morărescu, and Jiang-Wen Xiao
}

\begin{abstract}
This paper proposes the design of an event-triggered control strategy for consensus of interconnected two-time scales systems with structured uncertainty. The control design under consideration ensures also that consensus is achieved with an overall guaranteed cost. Since each system involves processes evolving on both fast and slow time scales, two Zeno-free eventtriggered mechanisms are designed to independently decide the sampling and transmission instants for the slow and fast states respectively. As the first step, we design an event-triggering consensus protocol in the ideal/nominal case when the interconnected systems are not affected by uncertainties and the interactions happen over a fixed interaction network. Next, the results are extended in order to take into account structured uncertainties affecting the systems dynamics. At this step, we go further and we provide sufficient conditions for event-triggering consensus with a guaranteed overall cost. Finally, two numerical examples are provided to demonstrate the effectiveness of the proposed theoretical results.
\end{abstract}

Index Terms-Event-triggered control, interconnected systems, two-time-scale, guaranteed cost.

\section{INTRODUCTION}

$\mathbf{T}$ HE last decades witnessed an increasing attention given to cooperative control of interconnected systems. This is certainly due to its numerous applications including mobile robots [1], monitoring control [2], biology [3], traffic flow [4] or opinion dynamics [5]. Consensus problem is the most popular problem in cooperative control of interconnected systems. Consensus protocols aim at driving each system towards an agreement relying on the information exchanged locally among neighbors.

Traditionally, the consensus protocols for continuous time dynamics are mostly designed to be applied continuously. Nevertheless, digital implementation of the controller is required in most of the applications. This means that control signals can be updated only at particular discrete time instants corresponding to interaction/communication times. Moreover, in order to extend the life cycle of devices we are often interested to minimize the number of interactions and implicitly the number

This work is supported by the National Natural Science Foundation of China (61773172), the National Key Research and Development Program, and the Fundamental Research Funds for the Central Universities (2018KFYYXJJ119). The work of I.C. Morărescu has been partially funded by ANR under grant HANDY ANR-18-CE40-0010.

Y. Lei, Y.W. Wang and J.W. Xiao are with School of Artificial Intelligence and Automation, Huazhong University of Science and Technology, Wuhan, 430074, China and with Key Laboratory of Image Processing and Intelligent Control(Huazhong University of Science and Technology), Ministry of Education, Wuhan, 430074, China. I.C. Morărescu is with Université de Lorraine, CNRS, CRAN, F-54000 Nancy, France. E-mail: wangyw@hust.edu.cn. of control updates. There are many effective way to save the communication and control resources, for example, impulsive control [6], periodic sampling control [7], intermittent control [8], event-triggered control [9]-[11] and the combination of these strategies [12]. Among all these strategies, eventtriggered strategy is very attractive since each system only needs to update control actuation and communicate with neighbors when a pre-defined event is triggered. A distributed event-triggered control strategy requires a distributed eventtriggered controller and a distributed Zeno-free event-triggered mechanism. In [13], [14], two distributed event-triggered control strategy are proposed to achieve the consensus of the interconnected systems with single-integrator dynamic. Then, the event-triggered consensus problem is further studied for the interconnected systems with double-integrator [15] and general linear dynamics [16]-[19]. In [20], a self-triggered scheme is further proposed to avoid the continuous monitoring of the neighbors states and achieve the output consensus of heterogeneous linear interconnected systems. It is noteworthy that all these studies consider only dynamics evolving on one time scale while in many practical applications the dynamics evolves on two time scales.

Biological systems [21], chemical reactions [22], power systems [23], [24] involve both slow and fast processes leading to dynamics that are mathematically described as two-time scales systems. Feedback design for such two time-scales systems (TTSSs)is often subject to high dimensionality and is numerical ill-conditioned. Consequently, it is interesting, yet challenging, to consider the problem of control design for consensus of interconnected TTSSs. The stabilization problem of centralized TTSSs has been widely studied [25]-[28], but the corresponding results on the consensus of interconnected TTSSs are relatively few. In [29], the time scale decomposition method is utilized to achieve the consensus of interconnected TTSSs. In [30], time-varying delay and switching interaction topology are further considered for the bipartite consensus of interconnected TTSSs. However, the Laplacian matrix associating with the interaction topology is required to be known in [29], [30]. In [31], the proposed consensus control protocol for the interconnected TTSSs is independent of the Laplacian matrix and only depends on the size of the interaction network. A practical limitation of the designs in [29]-[31] is that the consensus algorithms have to be continuously applied. To overcome this limitation, in [32] the authors designed an event-triggered control strategy for synchronization of a class of nonlinear TTSSs in which the control inputs acts 
independently on each component (i.e. the coefficient of the input is the identity matrix). Beside that, as pointed out in [33], energy aware strategies need to guaranty an overall synchronization cost while saving communication resources.

The main contribution of this paper is threefold. First, we extend the results in [31] by proposing an event-triggering protocol to synchronize TTSSs with a guaranteed cost. Second, we analyze the closed-loop dynamics through Lyapunov techniques and show that event-triggering mechanisms for slow and fast states are Zeno free and not synchronized. Third, the results are proven in the wider framework of TTSSs with structured uncertainties. This last feature renders the results implementable in real applications in which the agents are slightly different although they are supposed to be identical.

The rest of the paper is organized as follows. Some preliminaries of algebraic graph theory and guaranteed-cost consensus are introduced in Section II. The analysis of the guaranteed-cost consensus problem of linear interconnected TTSSs under fixed interaction topology is detailed in Section III. In this section we first analyze the synchronization for nominal/identical systems and then we extend the results to the case of dynamics affected by uncertainties. Two illustrative examples are presented in Section IV. Conclusion is drawn in Section V.

Notation. $\mathbb{R}^{m \times n}$ denotes the set of $m \times n$ real matrices. We write $P>0$ to precise that a real symmetric matrix $P$ is positive definite. $\lambda_{\min }(P)$ and $\lambda_{\max }(P)$ represent the minimum and the maximum eigenvalue, respectively. $\|\cdot\|$ denotes the Euclidean norm for vectors or the induced 2-norm for matrices. $\otimes$ stands for Kronecker product. The notation $\operatorname{diag}\left(d_{1}, \ldots, d_{N}\right)$ denotes the diagonal matrix with diagonal elements $d_{1}, \ldots, d_{N}$.

\section{Problem Formulation And Preliminaries} A. Graph Theory

A graph $\mathcal{G}=(\mathcal{V}, \mathcal{E}, \mathcal{A})$ is defined by the vertex-set $\mathcal{V}=$ $\{1,2, \ldots, N\}$, edge-set $\mathcal{E} \subset \mathcal{V} \times \mathcal{V}$ and adjacency matrix $\mathcal{A} \in$ $\mathbb{R}^{N \times N}$. System $i$ obtains information from system $j$ if and only if $\{j, i\} \in \mathcal{E}$. The adjacency matrix is defined as $\mathcal{A}=$ $\left(a_{i j}\right)_{N \times N}$, with $a_{i j}>0$ if and only if $\{j, i\} \in \mathcal{E}$, otherwise, $a_{i j}=0$. It is assumed that $a_{i i}=0$. A graph $\mathcal{G}=(\mathcal{V}, \mathcal{E}, \mathcal{A})$ is undirected if $a_{i j}=a_{j i}$, for $\forall i, j \in \mathcal{V}$. A sequence of distinct adjacent vertices starting with $i$ and ending with $j$ is called a path from $i$ to $j$. If there is a path between any two nodes of the graph $\mathcal{G}$, then $\mathcal{G}$ is called connected. The Laplacian matrix $L=\left(l_{i j}\right)_{N \times N}$ of graph $\mathcal{G}$ is defined as $l_{i j}=-a_{i j}, i \neq j$ and $l_{i i}=\sum_{k=1, k \neq i}^{N} a_{i k}$. As in [31] we impose the following Assumption.

Assumption 1. The interaction topology $\mathcal{G}$ is undirected and connected. All the non-zero weight $a_{i j} \neq 0$ of the associated weighted Laplacian matrix are within the interval $\left[a_{m}, a_{M}\right]$ with $a_{M} \geq a_{m}>0$.

Under Assumption 1 the following result holds.

Lemma 1 ( [31]). Let an undirected graph $\mathcal{G}$ satisfy Assumption 1 and let $0=\lambda_{1}<\lambda_{2} \leq \ldots \leq \lambda_{N}$ be the eigenvalues of the corresponding Laplacian matrix $L$. A rough lower-bound on $\lambda_{2}$, independent of $\mathcal{G}$, is $\lambda^{*}=\frac{a_{m}^{2}}{2(N-1) N^{2}}$. Therefore, it can be obtained that

$$
\lambda^{*}<\lambda_{2} \leq \ldots \lambda_{N}<N \cdot a_{M} \triangleq \lambda^{\circ} .
$$

There exists an orthonormal matrix $T \in \mathbb{R}^{N \times N}$ (i.e. $T T^{T}=$ $\left.T^{T} T=I_{N}\right)$ such that $T L T^{T}=D=\operatorname{diag}\left(\lambda_{1}, \lambda_{2}, \ldots, \lambda_{N}\right)$.

\section{B. Problem Description}

Consider the following interconnected systems composed of $N$ uncertain linear TTSSs,

$$
\left(\begin{array}{c}
\dot{x}_{i}(t) \\
\varepsilon \dot{z}_{i}(t)
\end{array}\right)=\left(A+\Xi_{i}\right)\left(\begin{array}{c}
x_{i}(t) \\
z_{i}(t)
\end{array}\right)+B u_{i}(t),
$$

where $i=1, \ldots, N, x_{i}(t) \in \mathbb{R}^{n_{x}}$ and $z_{i}(t) \in \mathbb{R}^{n_{z}}$ are the slow and fast state vectors, respectively, $\varepsilon$ is a small positive parameter defining the time-scale separation between slow and fast dynamics, $u_{i}(t) \in \mathbb{R}^{p}$ is the control input vector, $A=\left(\begin{array}{ll}A_{11} & A_{12} \\ A_{21} & A_{22}\end{array}\right), B=\operatorname{col}\left(B_{1}, B_{2}\right), A_{i j}, B_{i}, i, j=1,2$, are the known constant matrices with appropriate dimensions, $\Xi_{i}=\sum_{k=1}^{m_{i}} q_{i k} \Xi_{i k}$ represents the structured uncertainty with $q_{i k}$ uncertain parameters and $\Xi_{i k}$ known constant matrices.

Definition 1. The consensus of linear interconnected TTSSs (1) is achieved, if for any given admissible initial state $x_{i}(0), z_{i}(0), \lim _{t \rightarrow \infty}\left\|x_{i}(t)-x_{j}(t)\right\|=0$, $\lim _{t \rightarrow \infty}\left\|z_{i}(t)-z_{j}(t)\right\|=0, \forall i, j=1, \ldots, N$.

For each system $i,\left\{t_{k}^{1 i}\right\}$ for $x_{i}$ and $\left\{t_{k}^{2 i}\right\}$ for $z_{i}, k \in$ $0,1,2, \ldots$, are two increasing sequences of triggering instants at which system $i$ will respectively update the states $x_{i}$ and $z_{i}$ and send to its neighbors. Let $t_{0}^{1 i}=t_{0}^{2 i}=0$, for $i \in \mathcal{V}$. Define $\hat{x}_{i}(t)=x_{i}\left(t_{k_{1 i}}^{1 i}\right)$ and $\hat{z}_{i}(t)=z_{i}\left(t_{k_{2 i}}^{2 i}\right)$ as the latest sampled slow and fast states values of system $i$ at time $t$. The distributed event-triggered controller is designed in the following form:

$$
u_{i}(t)=K_{1} \sum_{j=1}^{N} a_{i j}\left(\hat{x}_{j}(t)-\hat{x}_{i}(t)\right)+K_{2} \sum_{j=1}^{N} a_{i j}\left(\hat{z}_{j}(t)-\hat{z}_{i}(t)\right) .
$$

Define the measurement errors of slow and fast states as follows

$$
e_{1 i}(t)=\hat{x}_{i}(t)-x_{i}(t), e_{2 i}(t)=\hat{z}_{i}(t)-z_{i}(t) .
$$

The sequences $\left\{t_{k}^{1 i}\right\},\left\{t_{k}^{2 i}\right\}$ for $x_{i}$ and $z_{i}$ are determined by the event-triggered mechanism designed in the following form

$$
\begin{aligned}
& t_{k+1}^{1 i}=\inf \left\{t>t_{k}^{1 i} \mid g\left(e_{1 i}(t), q_{1 i}(t), \delta_{1 i}(t)\right)=0\right\}, \\
& t_{k+1}^{2 i}=\inf \left\{t>t_{k}^{2 i} \mid g\left(e_{2 i}(t), q_{2 i}(t), \delta_{2 i}(t)\right)=0\right\},
\end{aligned}
$$

where $g(\cdot)$ is a nonlinear function to be designed, $q_{1 i}(t)=$ $\sum_{j=1}^{N} a_{i j}(t)\left(\hat{x}_{i}(t)-\hat{x}_{j}(t)\right), q_{2 i}(t)=\sum_{j=1}^{N} a_{i j}(t)\left(\hat{z}_{i}(t)-\hat{z}_{j}(t)\right)$, $\delta_{1 i}(t)$ and $\delta_{2 i}(t)$ are two positive smooth functions and square integrable over $t \in[0, \infty)$.

Remark 1. For each system $i$, the event-triggered controller (2) only updates when the event-triggered mechanisms (3) 
and (4) of itself or neighbors are triggered. From the eventtriggered mechanisms (3) and (4), it can be obtained that the triggering instants for the slow and fast states are asynchronous and independently generated. The functions $\delta_{1 i}$ and $\delta_{2 i}$ in (3) and (4) are introduced to exclude the Zeno behavior. The necessity of $\delta_{1 i}$ and $\delta_{2 i}$ being in such form will be clear from the theoretical analysis.

To convert the consensus problem of the interconnected TTSSs (1) into a stabilization problem, the following state and input transformations are performed

$$
\begin{aligned}
& \tilde{x}(t)=\left(T \otimes I_{n_{x}}\right) x(t), \tilde{z}(t)=\left(T \otimes I_{n_{z}}\right) z(t), \\
& \tilde{u}(t)=\left(T \otimes I_{q}\right) u(t), \tilde{e}_{1}(t)=\left(T \otimes I_{n_{x}}\right) e_{1}(t), \\
& \tilde{e}_{2}(t)=\left(T \otimes I_{n_{z}}\right) e_{2}(t),
\end{aligned}
$$

where $T$ is defined in Lemma 1 and $x=\operatorname{col}\left(x_{1}, \ldots, x_{N}\right)$, $z=\operatorname{col}\left(z_{1}, \ldots, z_{N}\right), u=\operatorname{col}\left(u_{1}, \ldots, u_{N}\right), e_{1}=$ $\operatorname{col}\left(e_{11}, \ldots, e_{1 N}\right), e_{2}=\operatorname{col}\left(e_{21}, \ldots, e_{2 N}\right)$. Then, the control law $\tilde{u}(t)$ can be rewritten as follows,

$$
\begin{aligned}
\tilde{u}(t)= & -\left(T L \otimes K_{1}\right) x(t)-\left(T L \otimes K_{2}\right) z(t) \\
& -\left(T L \otimes K_{1}\right) e_{1}(t)-\left(T L \otimes K_{2}\right) e_{2}(t) \\
= & -\left(T L T^{T} \otimes K_{1}\right) \tilde{x}(t)-\left(T L T^{T} \otimes K_{2}\right) \tilde{z}(t) \\
& -\left(T L T^{T} \otimes K_{1}\right) \tilde{e}_{1}(t)-\left(T L T^{T} \otimes K_{2}\right) \tilde{e}_{2}(t) \\
= & -\left[\left(D \otimes K_{1}\right) \tilde{x}(t)+\left(D \otimes K_{2}\right) \tilde{z}(t)\right] \\
& -\left[\left(D \otimes K_{1}\right) \tilde{e}_{1}(t)+\left(D \otimes K_{2}\right) \tilde{e}_{2}(t)\right] .
\end{aligned}
$$

Denote $\tilde{u}=\operatorname{col}\left(\tilde{u}_{1}, \ldots, \tilde{u}_{N}\right)$. Then, for $i=1, \ldots, N$,

$$
\tilde{u}_{i}(t)=-\lambda_{i} K_{1}\left(\tilde{x}_{i}(t)+\tilde{e}_{1 i}(t)\right)-\lambda_{i} K_{2}\left(\tilde{z}_{i}(t)+\tilde{e}_{2 i}(t)\right) .
$$

Denote $\tilde{x}=\operatorname{col}\left(\tilde{x}_{1}, \ldots, \tilde{x}_{N}\right), \tilde{z}=\operatorname{col}\left(\tilde{z}_{1}, \ldots, \tilde{z}_{N}\right)$, $\tilde{e}_{1}=\operatorname{col}\left(\tilde{e}_{11}, \ldots, \tilde{e}_{1 N}\right), \tilde{e}_{2}=\operatorname{col}\left(\tilde{e}_{21}, \ldots, \tilde{e}_{2 N}\right), \Xi=$ $\operatorname{diag}\left\{\Xi_{1}, \ldots, \Xi_{N}\right\}$ and $\tilde{\Xi}=\operatorname{col}\left(\tilde{\Xi}_{1}, \ldots, \tilde{\Xi}_{N}\right)=(T \otimes$ $\left.I_{n_{x}+n_{z}}\right) \Xi\left(T^{T} \otimes I_{n_{x}+n_{z}}\right)$. The closed-loop system (1) and (2) can be decoupled into $n$ independent TTSSs, for $i=1, \ldots, n$,

$$
\left(\begin{array}{c}
\dot{\tilde{x}}_{i} \\
\varepsilon \dot{\tilde{z}}_{i}
\end{array}\right)=\left(\left(\begin{array}{cc}
\Lambda_{11}^{i} & \Lambda_{12}^{i} \\
\Lambda_{21}^{i} & \Lambda_{22}^{i}
\end{array}\right)+\tilde{\Xi}_{i}\right)\left(\begin{array}{c}
\tilde{x}_{i} \\
\tilde{z}_{i}
\end{array}\right)-\lambda_{i} B K\left(\begin{array}{c}
\tilde{e}_{1 i} \\
\tilde{e}_{2 i}
\end{array}\right),
$$

where $K=\operatorname{col}\left(K_{1}, K_{2}\right)$,

$$
\begin{aligned}
& \Lambda_{11}^{i}=A_{11}-\lambda_{i} B_{1} K_{1}, \Lambda_{12}^{i}=A_{12}-\lambda_{i} B_{1} K_{2}, \\
& \Lambda_{21}^{i}=A_{21}-\lambda_{i} B_{2} K_{1}, \Lambda_{22}^{i}=A_{22}-\lambda_{i} B_{2} K_{2} .
\end{aligned}
$$

It can be easily obtained that, when the event-triggered control law (2) achieves the stabilization of system (7) for $i=2, \ldots, N$, the consensus problem of system (1) is also solved.

Consider the following global cost associated with consensus of interconnected TTSSs (1):

$$
\begin{aligned}
J= & \int_{0}^{\infty} x(t)^{T}\left(L \otimes I_{n_{x}}\right) x(t)+z(t)^{T}\left(L \otimes I_{n_{z}}\right) z(t) \\
& +u(t)^{T}\left(I_{p} \otimes R\right) u(t) d t,
\end{aligned}
$$

where $R \in \mathbb{R}^{q \times q}$ is a positive definite matrix that penalizes the control effort required for consensus.

Definition 2. The guaranteed-cost consensus of linear interacted TTSSs (1) is said to be achieved, if there exists a bounded
$J^{*}$ such that the consensus is achieved and $J \leq J^{*}$, where $J^{*}$ is said to be a guaranteed cost.

The main goal of this paper is to characterize the feedback controllers (2) together with the event-triggered mechanism (3) and (4) such that the guaranteed-cost consensus of the interconnected systems (1) can be achieved.

\section{MAIN RESULT}

In this section, we firstly present an event-triggered control laws to achieve the guaranteed-cost consensus of the interconnected nominal TTSSs with fixed undirected topology. Then, the results are extended to take into account structured uncertainty on the systems dynamics.

\section{A. Analysis of nominal interconnected TTSSs}

In this subsection, we consider the following interconnected nominal TTSS:

$$
\left\{\begin{array}{l}
\dot{x}_{i}(t)=A_{11} x_{i}(t)+A_{12} z_{i}(t)+B_{1} u_{i}(t), \\
\varepsilon \dot{z}_{i}(t)=A_{21} x_{i}(t)+A_{22} z_{i}(t)+B_{2} u_{i}(t),
\end{array}\right.
$$

where $i=1, \ldots, N$. Unlike (1), nominal TTSSs (9) consider all the agents are driven by identical dynamics. Suppose the interaction topology satisfies Assumption 1.

To conduct the Chang transformation, the following assumptions and lemma are necessary.

Assumption 2. The matrix $A_{22}$ is invertible.

Assumption 3. The pairs $\left(A_{0}, B_{0}\right)$ and $\left(A_{22}, B_{2}\right)$ are stabilizable, where $A_{0}=A_{11}-A_{12} A_{22}^{-1} A_{21}, B_{0}=B_{1}-A_{12} A_{22}^{-1} B_{2}$.

Lemma 2 ( [20]). For any stabilizable pair $(A, B)$, there always exists a unique symmetric matrix $P>0$ satisfying the following algebraic Riccati equation:

$$
P A+A^{T} P-2 \mu_{1} P B B^{T} P+\mu_{2} I_{n}=0,
$$

where $\mu_{1}, \mu_{2}$ are two positive constant.

Remark 2. Assumptions 2, 3 are standard, they have been also used in [31] to ensure that stabilization of system (9) can be achieved. By Lemma 2, there exist $P_{1}=P_{1}^{T}>0$, $P_{2}=P_{2}^{T}>0, Q_{1}>0, Q_{2}>0$ such that

$$
\begin{gathered}
P_{1} A_{0}+A_{0}^{T} P_{1}-2 \frac{\lambda^{*}}{\lambda^{\circ}} P_{1} B_{0} B_{0}^{T} P_{1}+Q_{1}=0, \\
P_{2} A_{22}+A_{22}^{T} P_{2}-2 \lambda^{*} P_{2} B_{2} B_{2}^{T} P_{2}+Q_{2}=0 .
\end{gathered}
$$

Thus, there exist $K_{0}=B_{0}^{T} P_{1}, K_{2}=B_{2}^{T} P_{2}$, such that,

$$
\begin{gathered}
\left(A_{0}-\frac{\lambda_{i}}{\lambda^{\circ}} B_{0} K_{0}\right)^{T} P_{1}+P_{1}\left(A_{0}-\frac{\lambda_{i}}{\lambda^{\circ}} B_{0} K_{0}\right)+Q_{1} \leq 0, \\
\left(A_{22}-\lambda_{i} B_{2} K_{2}\right)^{T} P_{2}+P_{2}\left(A_{22}-\lambda_{i} B_{2} K_{2}\right)+Q_{2} \leq 0,
\end{gathered}
$$

for $i=2, \ldots, N$, which means the matrices $A_{0}-\frac{\lambda_{i}}{\lambda^{\circ}} B_{0} K_{0}$ and $A_{22}-\lambda_{i} B_{2} K_{2}$ are all Hurwitz. The reason of $P_{1}$ and $P_{2}$ being designed to satisfy (11) and (12) will be derived in the following theoretical analysis.

Now, the Chang transformation is ready to be given. Define

$T_{i c}=\left(\begin{array}{cc}I_{n_{x}} & \varepsilon H_{i} \\ -L_{i} & I_{n_{z}}-\varepsilon L_{i} H_{i}\end{array}\right), T_{i c}^{-1}=\left(\begin{array}{cc}I_{n_{x}}-\varepsilon H_{i} L_{i} & -\varepsilon H_{i} \\ L_{i} & I_{n_{z}}\end{array}\right)$, 
where the matrices $L_{i}$ and $H_{i}$ satisfy follow equations,

$$
\begin{aligned}
& \Lambda_{21}^{i}-\Lambda_{22}^{i} L_{i}+\varepsilon L_{i} \Lambda_{11}^{i}-\varepsilon L_{i} \Lambda_{12}^{i} L_{i}=0, \\
& \Lambda_{12}^{i}-H_{i} \Lambda_{22}^{i}+\varepsilon \Lambda_{11}^{i} H_{i}-\varepsilon \Lambda_{12}^{i} L_{i} H_{i}-\varepsilon H_{i} L_{i} \Lambda_{12}^{i}=0 .
\end{aligned}
$$

Then Chang transformation is performed

$$
\left(\begin{array}{c}
\tilde{x}_{i s}(t) \\
\tilde{z}_{i f}(t)
\end{array}\right)=T_{i c}^{-1}\left(\begin{array}{c}
\tilde{x}_{i}(t) \\
\tilde{z}_{i}(t)
\end{array}\right),
$$

where $\tilde{x}_{i s}(t), \tilde{z}_{i f}(t)$ are pure slow and pure fast state variables. As $\Lambda_{22}^{i}=A_{22}-\lambda_{i} B_{2} K_{2}$ is Hurwitz, it is non-singular, equations (15) have approximate solution. Then, the following system can be obtained, for $i=2, \ldots, N$,

$$
\left(\begin{array}{c}
\dot{\tilde{x}}_{i s}(t) \\
\dot{\tilde{z}}_{i f}(t)
\end{array}\right)=A_{i D}\left(\begin{array}{c}
\tilde{x}_{i s}(t) \\
\tilde{z}_{i f}(t)
\end{array}\right)-B_{i D} K\left(\begin{array}{c}
\tilde{e}_{1 i}(t) \\
\tilde{e}_{2 i}(t)
\end{array}\right) .
$$

where $A_{i D}$ is a block diagonal matrix with

$$
\begin{aligned}
A_{i D} & =\left(\begin{array}{cc}
A_{i s}-\lambda_{i} B_{i s} K_{i s} & 0 \\
0 & \frac{A_{i f}-\lambda_{i} B_{i f} K_{2}}{\varepsilon}
\end{array}\right), B_{i D}=\left(\begin{array}{c}
\lambda_{i} B_{i s} \\
\frac{\lambda_{i} B_{i f}}{\varepsilon}
\end{array}\right), \\
A_{i s} & =A_{0}-\varepsilon A_{12} A_{22}^{-1} L_{i}\left(A_{11}-A_{12} L_{i}\right), \\
B_{i s} & =B_{0}-\varepsilon A_{12} A_{22}^{-1} B_{1}, K_{i s}=K_{1}-K_{2} L_{i}, \\
A_{i f} & =A_{22}+\varepsilon L_{i} A_{12}, B_{i f}=B_{2}+\varepsilon L_{i} B_{1} .
\end{aligned}
$$

From (16), it can be obtained that if the proposed control stabilizes systems (17), the stabilization of systems (7) is also achieved for $i=2, \ldots, n$. Thus, the consensus problem of (9) is converted to the stabilization problem of systems (17) for $i=2, \ldots, N$.

Before giving the event-triggered control scheme, we firstly design the emulation control scheme for the consensus of interconnected TTSSs (9), where $e_{1 i} \equiv 0, e_{2 i} \equiv 0, i=1, \ldots, N$.

Theorem 1. Suppose that Assumptions 1-3 hold. There exist two positive definite symmetric matrices $P_{1}$ and $P_{2}$ satisfying (11) and (12). Let $K_{0}=B_{0}^{T} P_{1}, K_{2}=B_{2}^{T} P_{2}$. Then, there exists $\bar{\varepsilon}>0$ such that for all $\varepsilon \in(0, \bar{\varepsilon}]$, the interconnected TTSSs (9) achieve consensus with the following timecontinuous controller

$$
u_{i}(t)=K_{1} \sum_{j=1}^{N} a_{i j}\left(x_{j}(t)-x_{i}(t)\right)+K_{2} \sum_{j=1}^{N} a_{i j}\left(z_{j}(t)-z_{i}(t)\right) .
$$

with $K_{1}=\left(\frac{1}{\lambda^{\circ}}-K_{2} A_{22}^{-1} B_{2}\right) K_{0}+K_{2} A_{22}^{-1} A_{21}$.

Proof. As $K_{1}=\left(\frac{1}{\lambda^{\circ}}-K_{2} A_{22}^{-1} B_{2}\right) K_{0}+K_{2} A_{22}^{-1} A_{21}$, it can be obtained that from the first equation in (15)

$$
\begin{aligned}
L_{i}= & \left(A_{22}-\lambda_{i} B_{2} K_{2}\right)^{-1}\left(A_{21}-\lambda_{i} B_{2} K_{1}\right)+O(\varepsilon) \\
= & \left(A_{22}-\lambda_{i} B_{2} K_{2}\right)^{-1}\left(A_{21}-\lambda_{i} B_{2} K_{2} A_{22}^{-1} A_{21}\right)+O(\varepsilon) \\
& -\left(A_{22}-\lambda_{i} B_{2} K_{2}\right)^{-1} B_{2}\left(\frac{\lambda_{i}}{\lambda^{\circ}}-\lambda_{i} K_{2} A_{22}^{-1} B_{2}\right) K_{0} \\
= & \left(A_{22}-\lambda_{i} B_{2} K_{2}\right)^{-1}\left(A_{22}-\lambda_{i} B_{2} K_{2}\right) A_{22}^{-1} A_{21}+O(\varepsilon) \\
& -\left(A_{22}-\lambda_{i} B_{2} K_{2}\right)^{-1}\left(\frac{\lambda_{i}}{\lambda^{\circ}} A_{22}-\lambda_{i} B_{2} K_{2}\right) A_{22}^{-1} B_{2} K_{0} \\
= & A_{22}^{-1} A_{21}-A_{22}^{-1} B_{2} K_{0}+O(\varepsilon) \\
& -\left(\frac{\lambda_{i}}{\lambda^{\circ}}-1\right)\left(A_{22}-\lambda_{i} B_{2} K_{2}\right)^{-1} B_{2} K_{0} .
\end{aligned}
$$

Then

$$
\begin{aligned}
& K_{i s}=\frac{1}{\lambda^{\circ}} K_{0}+\left(\frac{\lambda_{i}}{\lambda^{\circ}}-1\right) K_{2}\left(A_{22}-\lambda_{i} B_{2} K_{2}\right)^{-1} B_{2} K_{0}+O(\varepsilon), \\
& A_{i s}-\lambda_{i} B_{i s} K_{i s}=A_{0}-\frac{\lambda_{i}}{\lambda^{\circ}} B_{0} K_{0}-\lambda_{i}\left(\frac{\lambda_{i}}{\lambda^{\circ}}-1\right) B_{0} K_{2} \times \\
& \quad\left(A_{22}-\lambda_{i} B_{2} K_{2}\right)^{-1} B_{2} K_{0}+O(\varepsilon), \\
& A_{i f}-\lambda_{i} B_{i f} K_{2}=A_{22}-\lambda_{i} B_{2} K_{2}+O(\varepsilon), \\
& B_{i s}=B_{0}+O(\varepsilon), B_{i f}=B_{2}+O(\varepsilon) .
\end{aligned}
$$

Define the following Lyapunov candidate

$$
V=\sum_{\mathrm{i}=2}^{N} \tilde{x}_{i s}^{T} P_{1} \tilde{x}_{i s}+\varepsilon \sum_{\mathrm{i}=2}^{N} \tilde{z}_{i f}^{T} P_{2} \tilde{z}_{i f} .
$$

Since $e_{1 i} \equiv 0, e_{2 i} \equiv 0, i=1, \ldots, N$. The time derivative of $V$ along the trajectories of (17) with controller (18) is

$$
\begin{aligned}
\dot{V}= & \sum_{\mathrm{i}=2}^{N}\left(\dot{\tilde{x}}_{i s}^{T} P_{1} \tilde{x}_{i s}+\tilde{x}_{i s}^{T} P_{1} \dot{\tilde{x}}_{i s}\right)+\varepsilon \sum_{\mathrm{i}=2}^{N}\left(\dot{\tilde{z}}_{i f}^{T} P_{2} \tilde{z}_{i f}+\tilde{z}_{i f}^{T} P_{2} \dot{\tilde{z}}_{i f}\right) \\
= & \sum_{\mathrm{i}=2}^{N} \tilde{x}_{i s}^{T}\left(\left(A_{i s}-\lambda_{i} B_{i s} K_{i s}\right)^{T} P_{1}+P_{1}\left(A_{i s}-\lambda_{i} B_{i s} K_{i s}\right)\right) \tilde{x}_{i s} \\
& +\sum_{\mathrm{i}=2}^{N} \tilde{z}_{i f}^{T}\left(\left(A_{i f}-\lambda_{i} B_{i f} K_{2}\right)^{T} P_{2}+P_{2}\left(A_{i f}-\lambda_{i} B_{i f} K_{2}\right)\right) \tilde{z}_{i f} \\
= & \sum_{\mathrm{i}=2}^{N} \tilde{x}_{i s}^{T}\left(\left(A_{0}-\frac{\lambda_{i}}{\lambda^{\circ}} B_{0} K_{0}\right)^{T} P_{1}+P_{1}\left(A_{0}-\frac{\lambda_{i}}{\lambda^{\circ}} B_{0} K_{0}\right)\right) \tilde{x}_{i s} \\
& +2 \sum_{\mathrm{i}=2}^{N}\left(\lambda_{i}-\frac{\lambda_{i}^{2}}{\lambda^{\circ}} \tilde{x}_{i s}^{T} P_{1} B_{0} K_{2}\left(A_{22}-\lambda_{i} B_{2} K_{2}\right)^{-1} B_{2} K_{0} \tilde{x}_{i s}\right. \\
& +\sum_{\mathrm{i}=2}^{N} \tilde{z}_{i f}^{T}\left(\left(A_{22}-\lambda_{i} B_{2} K_{2}\right)^{T} P_{2}+P_{2}\left(A_{22}-\lambda_{i} B_{2} K_{2}\right)\right) \tilde{z}_{i f} \\
& +\sum_{\mathrm{i}=2}^{N} \tilde{x}_{i s}^{T} O(\varepsilon) \tilde{x}_{i s}+\sum_{\mathrm{i}=2}^{N} \tilde{z}_{i f}^{T} O(\varepsilon) \tilde{z}_{i f} \\
\leq & \sum_{\mathrm{i}=2}^{N}\left(\lambda_{i}-\frac{\lambda_{i}^{2}}{\lambda^{\circ}}\right) \tilde{x}_{i s}^{T} P_{1} B_{0} K_{2}\left(A_{22}-\lambda_{i} B_{2} K_{2}\right)^{-1} B_{2} K_{0} \tilde{x}_{i s} \\
& -\sum_{\mathrm{i}=2}^{N} \tilde{x}_{i s}^{T}\left(Q_{1}+O(\varepsilon)\right) \tilde{x}_{i s}-\sum_{\mathrm{i}=2}^{N} \tilde{z}_{i f}^{T}\left(Q_{2}+O(\varepsilon)\right) \tilde{z}_{i f},
\end{aligned}
$$

where the last equation is deduced from (13) and (14). Furthermore, from (14), it can be obtained that $P_{2} A_{22}+A_{22}^{T} P_{2}-$ $2 \lambda_{i} P_{2} B_{2} K_{2} \leq 0, i=1, \ldots, n$. Thus, for $i=1, \ldots, n$,

$$
\begin{aligned}
& \tilde{x}_{i s}^{T} P_{1} B_{0} K_{2}\left(A_{22}-\lambda_{i} B_{2} K_{2}\right)^{-1} B_{2} K_{0} \tilde{x}_{i s} \\
= & \tilde{x}_{i s}^{T} K_{0}^{T} K_{2}\left(P_{2} A_{22}-\lambda_{i} P_{2} B_{2} K_{2}\right)^{-1} K_{2}^{T} K_{0} \tilde{x}_{i s} \\
= & \check{x}_{i s}^{T}\left(P_{2} A_{22}-\lambda_{i} P_{2} B_{2} K_{2}\right)^{T} \check{x}_{i s} \\
= & \check{x}_{i s}^{T}\left(\frac{1}{2} P_{2} A_{22}+\frac{1}{2} A_{22}^{T} P_{2}-\lambda_{i} P_{2} B_{2} K_{2}\right) \check{x}_{i s} \leq 0,
\end{aligned}
$$

where $\check{x}_{i s}=\left(P_{2} A_{22}-\lambda_{i} P_{2} B_{2} K_{2}\right)^{-T} K_{2}^{T} K_{0} \tilde{x}_{i s}$. Thus

$$
\dot{V} \leq-\sum_{\mathrm{i}=2}^{N} \tilde{x}_{i s}^{T}\left(Q_{1}+O(\varepsilon)\right) \tilde{x}_{i s}-\sum_{\mathrm{i}=2}^{N} \tilde{z}_{i f}^{T}\left(Q_{2}+O(\varepsilon)\right) \tilde{z}_{i f} .
$$

Then, there exists $\bar{\varepsilon}>0$ such that for all $0<\varepsilon \leq \bar{\varepsilon}$,

$$
\dot{V} \leq-\theta_{1} \sum_{\mathrm{i}=2}^{N} \tilde{x}_{i s}^{T} \tilde{x}_{i s}-\theta_{2} \sum_{\mathrm{i}=2}^{N} \tilde{z}_{i f}^{T} \tilde{z}_{i f}
$$


for some $\theta_{1}>0, \theta_{2}>0$. Then, from standard Lyapunov stability results, we have $\lim _{t \rightarrow \infty} \tilde{x}_{i s}=0, \lim _{t \rightarrow \infty} \tilde{z}_{i f}=0$, for $i=2, \ldots, n$. The consensus of interconnected TTSSs (9) is achieved.

Remark 3. It can be seen that the design of (11) and (12) for $P_{1}$ and $P_{2}$ is to ensure that $A_{i s}-\lambda_{i} B_{i s} K_{i s}, A_{i f}-\lambda_{i} B_{i f} K_{2}$ are all Hurwitz for $i=2, \ldots, N$, and hence (20) holds, which leads to the stability of (17). Moreover, (11) and (12) depend only on the size of the network, and so does the design of the control matrices $K_{1}$ and $K_{2}$.

Now a distributed event-triggered controller in form of (2) is ready to be designed. Unlike controller (18), system (17) needs to consider the impact of $\tilde{e}_{1 i}(t), \tilde{e}_{2 i}(t)$ in this case.

Let $\mu=\max _{\lambda_{i} \in\left[\lambda^{*}, \lambda^{\circ}\right]}\left\{\left\|T_{i c}\left(\lambda_{i}\right)\right\|^{2}\right\}, \underline{\lambda}=\min _{i=1,2}\left\{\lambda_{\min }\left(Q_{i}\right)\right\}$, $\gamma=\frac{4 \lambda^{\circ 2}}{\underline{\lambda}}\left(\left\|P_{1} B_{0} K\right\|^{2}+\left\|P_{2} B_{2} K\right\|^{2}\right), \beta_{1}=\frac{\lambda_{\min }\left(Q_{1}\right)}{8 \mu \gamma \lambda^{\circ 2}+2 \lambda_{\min }\left(Q_{1}\right) \lambda^{\circ 2}}$, $\beta_{2}=\frac{\lambda_{\min }\left(Q_{2}\right)}{8 \mu \gamma \lambda^{\circ 2}+2 \lambda_{\min }\left(Q_{2}\right) \lambda^{\circ 2}}, \alpha_{1}=\max _{i=1,2}\left\{\left\|K_{i}^{T} R K_{i}\right\|^{2}\right\} \cdot \alpha_{2}=$ $\frac{4 \mu}{\underline{\lambda}}\left(\lambda^{\circ}+\frac{\alpha_{1} \lambda^{\circ 2}(\underline{\lambda}+4 \mu \gamma)}{\mu \gamma}\right)$. Since $\delta_{i j}(t), i=1,2, j=1, \ldots, N$ are all square integrable over $t \in[0, \infty), 0<2 \beta_{1} \lambda^{\circ}<$ 1 , there exists a positive constant $\alpha_{3}$ such that $\alpha_{3} \geq$ $\int_{0}^{\infty} \frac{2 \gamma \alpha_{2}+8 \alpha_{1} \lambda^{\circ 2}}{1-2 \beta_{1} \lambda^{\circ 2}} \sum_{i=1}^{N}\left(\delta_{1 i}^{2}+\delta_{2 i}^{2}\right) d t>0$.

Theorem 2. Suppose that Assumptions 1-3 hold. There exists $\bar{\varepsilon}>0$ such that for all $\varepsilon \in(0, \bar{\varepsilon}]$, the consensus of the interconnected TTSSs (9) can be achieved by the controller (2), where the matrices $K_{1}, K_{2}$ have the same definition as in Theorem 1 and the triggering instants $t_{k+1}^{1 i}, t_{k+1}^{2 i}$ are defined by event-triggered mechanism (21) and (22) as follows,

$$
\begin{aligned}
& t_{k+1}^{1 i}=\inf _{t>t_{k}^{1 i}}\left\{t \in \mathbb{R} \mid\left\|e_{1 i}\right\|^{2}=\beta_{1 i}\left\|q_{1 i}\right\|^{2}+\delta_{1 i}^{2}\right\}, \\
& t_{k+1}^{2 i}=\inf _{t>t_{k}^{2 i}}\left\{t \in \mathbb{R} \mid\left\|e_{2 i}\right\|^{2}=\beta_{2 i}\left\|q_{2 i}\right\|^{2}+\delta_{2 i}^{2}\right\},
\end{aligned}
$$

where $q_{1 i}, q_{2 i}, \delta_{1 i}, \delta_{2 i}$ have the same definition as in (3) and (4), $0 \leq \beta_{1 i} \leq \beta_{1}$ and $0 \leq \beta_{2 i} \leq \beta_{2}$. Furthermore, no system exhibits the Zeno behavior and a guaranteed cost $\beta=$ $\alpha_{2} V_{0}+\alpha_{3}$ is achieved for interconnected TTSSs (9), where $V_{0}=\sum_{\mathrm{i}=2}^{N} \tilde{x}_{i s}^{T}(0) P_{1} \tilde{x}_{i s}(0)+\varepsilon \sum_{\mathrm{i}=2}^{N} \tilde{z}_{i f}^{T}(0) P_{2} \tilde{z}_{i f}(0)$.

Proof. Define $\tilde{E}_{i}=\operatorname{col}\left(\tilde{e}_{1 i}, \tilde{e}_{2 i}\right)$. As in the proof of Theorem 1 and by considering the effect of $\tilde{e}_{1 i}(t), \tilde{e}_{2 i}(t)$, it can be obtained that the time derivative of $V$ along the trajectories of (17) with controller (2) is

$$
\begin{aligned}
\dot{V}= & \sum_{\mathrm{i}=2}^{N}\left(\dot{\tilde{x}}_{i s}^{T} P_{1} \tilde{x}_{i s}+\tilde{x}_{i s}^{T} P_{1} \dot{\tilde{x}}_{i s}\right)+\varepsilon \sum_{\mathrm{i}=2}^{N}\left(\dot{\tilde{z}}_{i f}^{T} P_{2} \tilde{z}_{i f}+\tilde{z}_{i f}^{T} P_{2} \dot{\tilde{z}}_{i f}\right) \\
\leq & -\sum_{\mathrm{i}=2}^{N} \tilde{x}_{i s}^{T}\left(Q_{1}+O(\varepsilon)\right) \tilde{x}_{i s}-\sum_{\mathrm{i}=2}^{N} \tilde{z}_{i f}^{T}\left(Q_{2}+O(\varepsilon)\right) \tilde{z}_{i f} \\
& -2 \sum_{\mathrm{i}=2}^{N} \lambda_{i}\left(\tilde{x}_{i s}^{T} P_{1} B_{i s}+\tilde{z}_{i f}^{T} P_{2} B_{i f}\right) K \tilde{E}_{i} \\
= & -\sum_{\mathrm{i}=2}^{N} \tilde{x}_{i s}^{T}\left(Q_{1}+O(\varepsilon)\right) \tilde{x}_{i s}-\sum_{\mathrm{i}=2}^{N} \tilde{z}_{i f}^{T}\left(Q_{2}+O(\varepsilon)\right) \tilde{z}_{i f} \\
& -2 \sum_{\mathrm{i}=2}^{N} \lambda_{i}\left(\tilde{x}_{i s}^{T}\left(P_{1} B_{0}+O(\varepsilon)\right)+\tilde{z}_{i f}^{T}\left(P_{2} B_{2}+O(\varepsilon)\right)\right) K \tilde{E}_{i}
\end{aligned}
$$

$$
\begin{aligned}
\leq & -\sum_{\mathrm{i}=2}^{N} \tilde{x}_{i s}^{T}\left(Q_{1}-\alpha_{1} I_{n_{x}}\right) \tilde{x}_{i s}-\sum_{\mathrm{i}=2}^{N} \tilde{z}_{i f}^{T}\left(Q_{2}-\alpha_{2} I_{n_{z}}\right) \tilde{z}_{i f} \\
& +\sum_{i=2}^{N} \lambda_{i}^{2}\left(\frac{\left\|P_{1} B_{0} K\right\|^{2}}{\alpha_{1}}+\frac{\left\|P_{2} B_{2} K\right\|^{2}}{\alpha_{2}}\right) \tilde{E}_{i}^{T} \tilde{E}_{i} \\
& +\sum_{\mathrm{i}=2}^{N} \tilde{x}_{i s}^{T} O(\varepsilon) \tilde{x}_{i s}+\sum_{\mathrm{i}=2}^{N} \tilde{z}_{i f}^{T} O(\varepsilon) \tilde{z}_{i f} .
\end{aligned}
$$

From the event-triggered mechanism (21), we have

$$
\begin{aligned}
\left\|e_{1 i}\right\|^{2} & \leq \beta_{1}\left\|\sum_{j=1}^{N} a_{i j}\left(\hat{x}_{i}-\hat{x}_{j}\right)\right\|^{2}+\delta_{1 i}^{2} \\
& \leq 2 \beta_{1}\left\|\sum_{j=1}^{N} a_{i j}\left(e_{1 i}-e_{1 j}\right)\right\|^{2}+2 \beta_{1}\left\|\sum_{j=1}^{N} a_{i j}\left(x_{i}-x_{j}\right)\right\|^{2}+\delta_{1 i}^{2} .
\end{aligned}
$$

Then, we can obtain that

$$
\begin{aligned}
e_{1}^{T} e_{1} & \leq 2 \beta_{1}\left\|\left(L \otimes I_{n_{x}}\right) e_{1}\right\|^{2}+2 \beta_{1}\left\|\left(L \otimes I_{n_{x}}\right) x\right\|^{2}+\sum_{i=1}^{N} \delta_{1 i}^{2} \\
& \leq 2 \beta_{1} \lambda^{\circ 2}\left\|e_{1}\right\|^{2}+2 \beta_{1} \tilde{x}^{T}\left(D^{2} \otimes I_{n_{x}}\right) \tilde{x}+\sum_{i=1}^{N} \delta_{1 i}^{2} \\
& \leq 2 \beta_{1} \lambda^{\circ 2}\left\|e_{1}\right\|^{2}+2 \beta_{1} \lambda^{\circ 2} \sum_{i=2}^{N} \tilde{x}_{i}^{T} \tilde{x}_{i}+\sum_{i=1}^{N} \delta_{1 i}^{2} .
\end{aligned}
$$

Thus,

$$
e_{1}^{T} e_{1} \leq \frac{2 \beta_{1} \lambda^{\circ 2}}{1-2 \beta_{1} \lambda^{\circ}} \sum_{i=2}^{N} \tilde{x}_{i}^{T} \tilde{x}_{i}+\frac{1}{1-2 \beta_{1} \lambda^{\circ}} \sum_{i=1}^{N} \delta_{1 i}^{2} .
$$

As $\beta_{1}=\frac{\lambda_{\min }\left(Q_{1}\right)}{8 \mu \gamma \lambda^{\circ 2}+2 \lambda_{\min }\left(Q_{1}\right) \lambda^{\circ 2}}$, then

$$
e_{1}^{T} e_{1} \leq \frac{\lambda_{\min }\left(Q_{1}\right)}{4 \mu \gamma} \sum_{\mathrm{i}=2}^{N} \tilde{x}_{i}^{T} \tilde{x}_{i}+\frac{1}{1-2 \beta_{1} \lambda^{\circ}} \sum_{i=1}^{N} \delta_{1 i}^{2} .
$$

Similarly to (23), it can be obtained that

$$
e_{2}^{T} e_{2} \leq \frac{\lambda_{\min }\left(Q_{2}\right)}{4 \mu \gamma} \sum_{\mathrm{i}=2}^{N} \tilde{z}_{i}^{T} \tilde{z}_{i}+\frac{1}{1-2 \beta_{1} \lambda^{\circ}} \sum_{i=1}^{N} \delta_{2 i}^{2} .
$$

From (16)

$$
\begin{aligned}
\left\|\operatorname{col}\left(\tilde{x}_{i}, \tilde{z}_{i}\right)\right\|^{2} & =\left\|T_{i c} \operatorname{col}\left(\tilde{x}_{i s}, \tilde{z}_{i f}\right)\right\|^{2} \\
& \leq \mu\left\|\operatorname{col}\left(\tilde{x}_{i s}, \tilde{z}_{i f}\right)\right\|^{2}
\end{aligned}
$$

thus

$$
\left\|\tilde{x}_{i s}\right\|^{2}+\left\|\tilde{z}_{i f}\right\|^{2} \geq \frac{1}{\mu}\left(\left\|\tilde{x}_{i}\right\|^{2}+\left\|\tilde{z}_{i}\right\|^{2}\right)
$$

As $\sum_{i=1}^{N}\left(\tilde{e}_{1 i}^{T} \tilde{e}_{1 i}+\tilde{e}_{2 i}^{T} \tilde{e}_{2 i}\right)=\sum_{i=1}^{N}\left(e_{1 i}^{T} e_{1 i}+e_{2 i}^{T} e_{2 i}\right)$, let $\alpha_{1}=\alpha_{2}=$ $\frac{1}{4} \underline{\lambda}$, then

$$
\begin{aligned}
\dot{V} \leq & -\sum_{\mathrm{i}=2}^{N} \tilde{x}_{i s}^{T}\left(\frac{3 Q_{1}}{4}+O(\varepsilon)\right) \tilde{x}_{i s}-\sum_{\mathrm{i}=2}^{N} \tilde{z}_{i f}^{T}\left(\frac{3 Q_{2}}{4}+O(\varepsilon)\right) \tilde{z}_{i f} \\
& +\gamma \sum_{i=2}^{N} \tilde{E}_{i}^{T} \tilde{E}_{i} \\
\leq & -\sum_{\mathrm{i}=2}^{N} \tilde{x}_{i s}^{T}\left(\frac{Q_{1}}{2}+O(\varepsilon)\right) \tilde{x}_{i s}-\sum_{\mathrm{i}=2}^{N} \tilde{z}_{i f}^{T}\left(\frac{Q_{2}}{2}+O(\varepsilon)\right) \tilde{z}_{i f} \\
& +\frac{\gamma}{1-2 \beta_{1} \lambda^{\circ}} \sum_{i=1}^{N}\left(\delta_{1 i}^{2}+\delta_{2 i}^{2}\right)
\end{aligned}
$$




$$
\leq-\left(\frac{\lambda}{2 \mu}+O(\varepsilon)\right) \sum_{i=2}^{N}\left(\tilde{x}_{i}^{T} \tilde{x}_{i}+\tilde{z}_{i}^{T} \tilde{z}_{i}\right)+\frac{\gamma}{1-2 \beta_{1} \lambda^{\circ 2}} \sum_{i=1}^{N}\left(\delta_{1 i}^{2}+\delta_{2 i}^{2}\right) .
$$

Thus, there exists $\bar{\varepsilon}>0$ such that for all $0<\varepsilon \leq \bar{\varepsilon}$,

$$
\dot{V} \leq-\frac{\lambda}{4 \mu} \sum_{\mathrm{i}=2}^{N}\left(\tilde{x}_{i}^{T} \tilde{x}_{i}+\tilde{z}_{i}^{T} \tilde{z}_{i}\right)+\frac{\gamma}{1-2 \beta_{1} \lambda^{\circ 2}} \sum_{i=1}^{N}\left(\delta_{1 i}^{2}+\delta_{2 i}^{2}\right) .
$$

Then, Zeno behavior will be excluded. As $e_{1 i}=\hat{x}_{i}-x_{i}$, the upper right-hand Dini derivative of $\left\|e_{1 i}(t)\right\|^{2}$ along the trajectories of (9) are

$$
\begin{aligned}
D^{+}\left\|e_{1 i}\right\|^{2} & \leq 2\left\|e_{1 i}\right\|\left\|\dot{e}_{1 i}\right\| \\
& \leq 2\left\|e_{1 i}\right\|\left\|A_{11}\left(\hat{x}_{i}-e_{1 i}\right)+A_{12}\left(\hat{z}_{i}-e_{2 i}\right)+B_{1} u_{i}\right\| \\
& \leq \\
& \left(2\left\|A_{11}\right\|+\left\|A_{12}\right\|+1\right)\left\|e_{1 i}\right\|^{2}+\left\|A_{12}\right\|\left\|e_{2 i}\right\|^{2} \\
& +\left\|A_{11} \hat{x}_{i}+A_{12} \hat{z}_{i}+B_{1} u_{i}\right\|^{2} .
\end{aligned}
$$

Similarly, it can be obtained that

$$
\begin{aligned}
D^{+}\left\|e_{2 i}\right\|^{2} \leq & \left(\left\|A_{21}\right\|+2\left\|A_{22}\right\|+1\right)\left\|e_{2 i}\right\|^{2}+\left\|A_{21}\right\|\left\|e_{1 i}\right\|^{2} \\
& +\left\|A_{21} \hat{x}_{i}+A_{22} \hat{z}_{i}+B_{2} u_{i}\right\|^{2} .
\end{aligned}
$$

Proof by contradiction will be used to ensure that no system will exhibit Zeno behavior. Firstly, the Zeno behavior will be excluded for event-triggered mechanism (21). In order to seek a contradiction, suppose that $\lim _{k \rightarrow \infty} t_{k}^{1 i}=T_{1 i}<\infty$. Since $\delta_{1 i}(t), \delta_{2 i}(t), i=1, \ldots, N$, are square integrable over $t \in[0, \infty)$, from (9), (21), (22) and (25), it can be obtained that $e_{1 i}(t), e_{2 i}(t), \hat{x}_{i}(t), \hat{z}_{i}(t), u_{i}(t)$ are all bounded on $t \in\left(0, T_{1 i}\right]$. Moreover, $\delta_{1 i}$ is positive smooth function. Thus, there are two positive constants $\alpha_{4}, \underline{\delta}_{1 i}$, such that, for $t \in\left(0, T_{1 i}\right]$,

$$
D^{+}\left\|e_{1 i}(t)\right\|^{2} \leq \alpha_{4}, 0<\underline{\delta}_{1 i} \leq \delta_{1 i}(t) .
$$

From (21), it can be obtained that

$$
t_{k+1}^{1 i}-t_{k}^{1 i} \geq \frac{\beta_{1 i}\left\|q_{1 i}\left(t_{k+1}^{1 i}\right)\right\|^{2}+\delta_{1 i}^{2}\left(t_{k+1}^{1 i}\right)}{\alpha_{4}} .
$$

Then,

$$
t_{k}^{1 i}=t_{k}^{1 i}-t_{k-1}^{1 i}+\ldots+t_{1}^{1 i}-t_{0}^{1 i}+t_{0}^{1 i} \geq k \frac{\delta_{1 i}}{\alpha_{4}}+t_{0}^{1 i} .
$$

Thus, it can be obtained that $\lim _{k \rightarrow \infty} t_{k}^{1 i} \geq \lim _{k \rightarrow \infty} k \frac{\delta_{1 i}}{\alpha_{4}}+t_{0}^{1 i}=\infty$, which yields a contradiction. Therefore, $\lim _{k \rightarrow \infty} t_{k}^{1 i}$ will not be bounded. Therefore, the Zeno behavior will not happen for event-triggered mechanism (21). With a similar proof, it can also be obtained that the Zeno behavior will not happen for event-triggered mechanism (22).

Since $\delta_{1 i}(t), \delta_{2 i}(t), i=1, \ldots, N$, are square integrable over $t \in[0, \infty)$, according to (25), it can be obtained that $V(t)$ is bounded and integrable on $t \in[0, \infty)$. Then $\sum_{i=2}^{N}\left(\tilde{x}_{i}^{T} \tilde{x}_{i}+\tilde{z}_{i}^{T} \tilde{z}_{i}\right)$ is also bounded and integrable on $t \in[0, \infty)$. Then $\tilde{x}_{i}, \tilde{z}_{i}$, $\tilde{e}_{1 i}, \tilde{e}_{2 i}, i=2, \ldots, N$, are all bounded on $t \in[0, \infty)$. From (17), $\dot{\tilde{x}}_{i}, \dot{\tilde{z}}_{i}, i=2, \ldots, N$, are also bounded on $t \in[0, \infty)$. By Barbalat's lemma, it can be concluded that $\lim _{t \rightarrow \infty}\left\|\tilde{x}_{i}\right\|=0$, $\lim _{t \rightarrow \infty}\left\|\tilde{z}_{i}\right\|=0$, for $i=2, \ldots, N$. Thus, the consensus of the interconnected TTSSs (9) is achieved.

In the following, we will prove that synchronization of (9) is achieved with a guaranteed cost.
From (5) and (6), one obtains that

$$
\begin{aligned}
J= & \int_{0}^{\infty} x^{T}(t)\left(L \otimes I_{n_{x}}\right) x(t)+z^{T}(t)\left(L \otimes I_{n_{z}}\right) z(t) \\
& +u^{T}(t)\left(I_{p} \otimes R\right) u(t) d t \\
= & \int_{0}^{\infty} \tilde{x}^{T}(t)\left(D \otimes I_{n_{x}}\right) \tilde{x}(t)+\tilde{z}^{T}(t)\left(D \otimes I_{n_{z}}\right) \tilde{z}(t) \\
& +\tilde{u}^{T}(t)\left(I_{p} \otimes R\right) \tilde{u}(t) d t \\
\leq & \int_{0}^{\infty} \sum_{i=2}^{N} \lambda_{i}\left(\tilde{x}_{i}^{T}(t) \tilde{x}_{i}(t)+\tilde{z}_{i}^{T}(t) \tilde{z}_{i}(t)\right)+\tilde{u}_{i}^{T}(t) R \tilde{u}_{i}(t) d t \\
\leq & \int_{0}^{\infty} \sum_{i=2}^{N}\left(\lambda^{\circ}+4 \alpha_{1} \lambda^{\circ 2}\right)\left(\tilde{x}_{i}^{T}(t) \tilde{x}_{i}(t)+\tilde{z}_{i}^{T}(t) \tilde{z}_{i}(t)\right) \\
& +4 \alpha_{1} \lambda^{\circ 2}\left(e_{1 i}^{T}(t) e_{1 i}(t)+e_{2 i}^{T}(t) e_{2 i}(t)\right) d t .
\end{aligned}
$$

Then, from (23) and (24), we deduce that

$$
\begin{aligned}
J \leq & \int_{0}^{\infty} \sum_{i=2}^{N}\left(\lambda^{\circ}+4 \alpha_{1} \lambda^{\circ 2}\right)\left(\tilde{x}_{i}^{T}(t) \tilde{x}_{i}(t)+\tilde{z}_{i}^{T}(t) \tilde{z}_{i}(t)\right) \\
& +\sum_{i=1}^{N} 4 \alpha_{1} \lambda^{\circ 2}\left(e_{1 i}^{T}(t) e_{1 i}(t)+e_{2 i}^{T}(t) e_{2 i}(t)\right) d t \\
\leq & \int_{0}^{\infty} \sum_{i=2}^{N}\left(\lambda^{\circ}+\frac{\alpha_{1} \lambda^{\circ 2}(\underline{\lambda}+4 \mu \gamma)}{\mu \gamma}\right)\left(\tilde{x}_{i}^{T}(t) \tilde{x}_{i}(t)+\tilde{z}_{i}^{T}(t) \tilde{z}_{i}(t)\right) \\
& +\frac{4 \alpha_{1} \lambda^{\circ 2}}{1-2 \beta_{1} \lambda^{\circ 2}} \sum_{i=1}^{N}\left(\delta_{1 i}^{2}+\delta_{2 i}^{2}\right) d t \\
\leq & \int_{0}^{\infty}-\alpha_{2} \dot{V}(t)+\frac{\gamma \alpha_{2}+4 \alpha_{1} \lambda^{\circ 2}}{1-2 \beta_{1} \lambda^{\circ 2}} \sum_{i=1}^{N}\left(\delta_{1 i}^{2}+\delta_{2 i}^{2}\right) d t \\
\leq & \alpha_{2} V_{0}+\alpha_{3} .
\end{aligned}
$$

Thus, the consensus of TTSSs (9) is achieved with a global guaranteed cost $\alpha_{2} V_{0}+\alpha_{3}$.

Remark 4. There are several aspects needed to be highlighted for the event-triggered mechanism.

1) By using Chang Transformation, the states of systems (7) are decoupled as pure slow and fast states. Thus the event-triggered mechanism for slow states and fast states can be designed independently as (21) and (22).

2) Due to the coupling of the slow and fast states, the upper bound $\beta_{1}$ of the event-triggering parameters $\beta_{1 i}$ dependents not only on the solution of (13) but also on (14), and so does the upper bound $\beta_{2}$ of $\beta_{2 i}$.

3) From (28), it can be obtained that the selection of $\delta_{1 i}$ and $\delta_{2 i}, i=1, \ldots, N$, will not only affect the triggering instants but also the guaranteed cost. To exclude the Zeno behavior and achieve the consensus with a global guaranteed cost, the functions $\delta_{1 i}$ and $\delta_{2 i}, i=1, \ldots, N$, are designed independently as in (3) and (4), and the detail will be given in the feasibility analysis later.

Remark 5. For each system $i$, the event-triggered mechanisms (21) and (22) depend on sampling states of itself and its neighbors, the measurement error and the designed function $\delta_{1 i}$ and $\delta_{2 i}$. It is also feasible by setting $\beta_{1 i}=\beta_{2 i}=0$. In this way, the event-triggered mechanisms (21) and (22) depend 
only on the measurement error and the designed function $\delta_{1 i}$ and $\delta_{2 i}$, but the events will be triggered more frequently.

Remark 6. If $\delta_{1 i}(t)$ and $\delta_{2 i}(t)$ are designed to be zero as in [18], [20], then Zeno behavior would be hard to exclude since $\underline{\delta}_{1 i}=\underline{\delta}_{2 i}=0$. If $\delta_{1 i}(t)$ and $\delta_{2 i}(t)$ are designed to be a positive constant as in [34] or a positive constant plus a positive function as in [35], then $\sum_{i=1}^{N} \delta_{1 i}^{2}$ and $\sum_{i=1}^{N} \delta_{2 i}^{2}$ are not integrable on $t \in[0, \infty)$, and the consensus of interconnected TTSSs (9) cannot be achieved and the global cost $J$ is unbounded. Thus, in this paper, $\delta_{1 i}(t)$ and $\delta_{2 i}(t)$ are designed to be positive smooth functions and square integrable over $t \in[0, \infty)$, which can exclude Zeno behavior and achieve the consensus with a global guaranteed cost.

B. Analysis of interconnected TTSSs with structured uncertainty

In this subsection, the previous results are extended to solve the guaranteed-cost consensus for interconnected TTSSs (1) with structured uncertainty under fixed undirected topology.

Considering TTSSs (1) with the distributed event-triggered controller (2), when Assumption 2 holds, by the state and input transformation similar to (5) and (16), it can be obtained that

$$
\begin{aligned}
\left(\begin{array}{c}
\dot{\tilde{x}}_{i s}(t) \\
\dot{\tilde{z}}_{i f}(t)
\end{array}\right)= & \left(A_{i D}+T_{i c}^{-1} E^{-1} \tilde{\Xi}_{i} T_{i c}\right)\left(\begin{array}{c}
\tilde{x}_{i s}(t) \\
\tilde{z}_{i f}(t)
\end{array}\right) \\
& -B_{i D} K\left(\begin{array}{c}
\tilde{e}_{1 i}(t) \\
\tilde{e}_{2 i}(t)
\end{array}\right) .
\end{aligned}
$$

where $E=\operatorname{diag}\left(I_{n_{x}}, \varepsilon I_{n_{z}}\right)$.

Similarly, when Assumption 3 holds, there exist $P_{1}=P_{1}^{T}>$ $0, P_{2}=P_{2}^{T}>0, Q_{1}>0, Q_{2}>0$ such that

$$
\begin{gathered}
P_{1} A_{0}+A_{0}^{T} P_{1}-2 \frac{\lambda^{*}}{\lambda^{\circ}} P_{1} B_{0} B_{0}^{T} P_{1}+2 Q_{1}=0, \\
P_{2} A_{22}+A_{22}^{T} P_{2}-2 \lambda^{*} P_{2} B_{2} B_{2}^{T} P_{2}+2 Q_{2}=0 .
\end{gathered}
$$

Then, the results in the interconnected TTSSs with structured uncertainty are given.

Theorem 3. Suppose that Assumptions 1-3 hold. There exist two positive definite symmetric matrices $P_{1}$ and $P_{2}$ satisfying (30) and (31). Let $K_{0}=B_{0}^{T} P_{1}, K_{2}=B_{2}^{T} P_{2}$. Then, there exists $\bar{\varepsilon}>0$ such that for all $\varepsilon \in(0, \bar{\varepsilon}]$, the interconnected TTSSs (1) achieve consensus when using controller (2) together with Zeno-free event-triggered mechanism (21) and (22), if for $i=1, \ldots, N$

$$
\sum_{k=1}^{m_{i}} q_{i k}^{2} \leq \max _{\lambda \in\left[\lambda^{*}, \lambda^{\circ}\right]}\left\{\sigma_{\max }^{-1}\left(\sum_{k=1}^{m_{i}}\left(Q^{-\frac{1}{2}} \hat{\Xi}_{i k}(\lambda) Q^{-\frac{1}{2}}\right)^{2}\right)\right\},
$$

where $Q=\operatorname{diag}\left(Q_{1}, Q_{2}\right), \hat{\Xi}_{i k}(\lambda)=P T_{i c}^{-1}(\lambda) E^{-1} \Xi_{i k} T_{i c}(\lambda)+$ $T_{i c}^{T}(\lambda) \Xi_{i k}^{T} E^{-1} T_{i c}^{-T}(\lambda) P, P=\operatorname{diag}\left(P_{1}, \varepsilon P_{2}\right), K_{1}$ and the parameters in (21) and (22) have same definition in Theorem 2. Furthermore, consensus of interconnected TTSSs (1) is obtained with a guaranteed cost $\beta=\alpha_{2} V_{0}+\alpha_{3}$, where $\alpha_{2}$ and $\alpha_{3}$ has same definition in Theorem 2 .

Proof. As in the proof of Theorem 2, the time derivative of $V$ in (19) along the trajectories of (29) with the controller (2) and event-triggered mechanism (21), (22) is

$$
\begin{aligned}
\dot{V} \leq & -\frac{\underline{\lambda}}{4 \mu} \sum_{i=2}^{N}\left(\tilde{x}_{i}^{T} \tilde{x}_{i}+\tilde{z}_{i}^{T} \tilde{z}_{i}\right)+\frac{\gamma}{1-2 \beta_{1} \lambda^{\circ}} \sum_{i=1}^{N}\left(\delta_{1 i}^{2}+\delta_{2 i}^{2}\right) \\
& -\hat{\xi}_{i}^{T}\left(Q-P T_{i c}^{-1} E^{-1} \tilde{\Xi}_{i} T_{i c}-T_{i c}^{T} \tilde{\Xi}_{i}^{T} E^{-1} T_{i c}^{-T} P\right) \hat{\xi}_{i}
\end{aligned}
$$

where $\hat{\xi}_{i}=\operatorname{col}\left(\tilde{x}_{i s}, \tilde{z}_{i f}\right)$. Then, following [36], using the condition (32), it can be obtained that, for $i, j=1, \ldots, N$,

$$
Q-P T_{i c}^{-1} E^{-1} \Xi_{j} T_{i c}+T_{i c}^{T} \Xi_{j}^{T} E^{-1} T_{i c}^{-T} P \leq 0
$$

Denote $T_{i j}$ being the element in the $i$-th row and $j$-th column of $T$. Then $\tilde{\Xi}_{i}=\sum_{j=1}^{N} T_{i j}^{2} \Xi_{i}$. Since $T T^{T}=I, \sum_{j=1}^{N} T_{i j}^{2}=1$. Thus, it can be obtained that

$$
P T_{i c}^{-1} E^{-1} \tilde{\Xi}_{i} T_{i c}+T_{i c}^{T} \tilde{\Xi}_{i}^{T} E^{-1} T_{i c}^{-T} P \leq \sum_{j=1}^{N} T_{i j}^{2} Q=Q .
$$

Thus (25) can also be obtained. Then, following the proof of Theorem 2, it can be concluded that Zeno behavior is excluded, $\lim _{t \rightarrow \infty}\left\|\tilde{x}_{i}\right\|=0, \lim _{t \rightarrow \infty}\left\|\tilde{z}_{i}\right\|=0$, for $i=2, \ldots, N$, and $J \leq \alpha_{2} V_{0}^{\infty}+\alpha_{3}$. Thus, consensus of interconnected TTSSs (1) is achieved with a guaranteed cost $\beta=\alpha_{2} V_{0}+\alpha_{3}$.

Remark 7. Although we are not considering the consensus of heterogenous TTSSs, this section we are making an important step towards practical implementation of our results. Indeed, here we are allowing each dynamics to be slightly different from the others since the structured uncertainties are not the same for all systems. Thus, the coupling term of structured uncertainty has to be handled to achieve the consensus of interconnected TTSSs (1), which would bring the difficulties in stability analysis.

\section{ILlustrative EXAMPLE}

In this section, two examples are presented to illustrate the obtained results on the event-triggered consensus of the linear interconnected TTSSs.

Example 1: Consider the interconnected TTSSs containing three systems, the dynamic of each system is given by (9) with

$$
\begin{aligned}
& A_{11}=\left(\begin{array}{cc}
2.5 & -6 \\
-2 & 2
\end{array}\right), A_{12}=\left(\begin{array}{cc}
2 & 3 \\
0 & -2
\end{array}\right), B_{1}=\left(\begin{array}{l}
0.2 \\
0.1
\end{array}\right), \\
& A_{21}=\left(\begin{array}{ll}
0.5 & 2 \\
-1 & 1
\end{array}\right), A_{22}=\left(\begin{array}{cc}
-2 & 1 \\
0 & -1
\end{array}\right), B_{2}=\left(\begin{array}{l}
0.1 \\
0.1
\end{array}\right) .
\end{aligned}
$$

and singular perturbation parameter $\varepsilon=0.01$. Thus, the matrix $A_{22}$ is invertible. Assumption 2 holds. Meanwhile,

$$
\begin{aligned}
& A_{0}=A_{11}-A_{12} A_{22}^{-1} A_{21}=\left(\begin{array}{cc}
-1 & 0 \\
0 & 0
\end{array}\right), \\
& B_{0}=B_{1}-A_{12} A_{22}^{-1} B_{2}=\left(\begin{array}{c}
0.7 \\
-0.1
\end{array}\right) .
\end{aligned}
$$

The pairs $\left(A_{0}, B_{0}\right)$ and $\left(A_{22}, B_{2}\right)$ are stabilizable. Assumption 3 holds. For system $i, i=1,2,3$, denote its slow and fast states as $\left(x_{i 1}, x_{i 2}\right)$ and $\left(z_{i 1}, z_{i 2}\right)$, respectively. Let $R=I_{2}$, then

$$
\begin{aligned}
J= & \int_{0}^{\infty} x(t)^{T}\left(L \otimes I_{n_{x}}\right) x(t)+z(t)^{T}\left(L \otimes I_{n_{x}}\right) z(t) \\
& +u(t)^{T} u(t) d t .
\end{aligned}
$$


The initial condition of system is taken as

$$
\begin{aligned}
& \left(x_{11}(0), x_{12}(0), z_{11}(0), z_{12}(0)\right)=(-4,-3,2,5), \\
& \left(x_{21}(0), x_{22}(0), z_{21}(0), z_{22}(0)\right)=(-1,2,4,2), \\
& \left(x_{31}(0), x_{32}(0), z_{31}(0), z_{32}(0)\right)=(6,-3,2,3) .
\end{aligned}
$$

The interaction topology $\mathcal{G}$ is given in Figure 1, which is undirected and connected. Assumption 1 holds. Choose

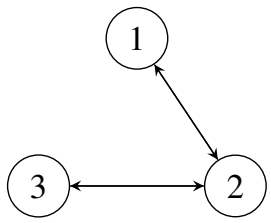

Fig. 1. The interaction topology $\mathcal{G}$.

$g_{m}=g_{M}=1$. From Lemma 1, it can be obtained that $\lambda^{*}=\frac{1}{36}$ and $\lambda^{\circ}=3$. Choose $Q_{1}=Q_{2}=10 I_{2}$, from (11) and (12), it can be obtained that

$$
P_{1}=\left(\begin{array}{cc}
4.9 & 1.415 \\
1.415 & 242.3
\end{array}\right), P_{2}=\left(\begin{array}{cc}
2.5 & 0.829 \\
0.829 & 5.817
\end{array}\right) \text {. }
$$

Then $K_{0}=B_{0}^{T} P_{1}=[3.29,-23.24], K_{1}=B_{2}^{T} P_{2}=$ $[0.33,0.67]$, and $K_{1}=\left(\frac{1}{\lambda^{\circ}}-K_{2} A_{22}^{-1} B_{2}\right) K_{0}+K_{2} A_{22}^{-1} A_{21}=$ $[2.172,-11.227]$. Based on Theorem 2, it can be obtained that $\beta 1=\beta 2=3.3 \times 10^{-5}$. Let $\beta_{i j}=\beta, \delta_{i j}=\delta$, for $i=1,2$, $j=1,2,3$. When $\beta=3.3 \times 10^{-5}$, and $\delta=2 e^{-0.5 t}$, simulation results of Theorem 1 and Theorem 2 show that consensus of both slow and fast states are achieved at a bounded cost, which confirms the effectiveness of Theorems 1 and 2 . The cost and numbers of triggering events with different $\beta, \delta$, are all shown in Table I, where $n_{i s}, n_{i f}$ are the numbers of triggering instants for $x_{i}$ and $z_{i}, i=1,2,3$, respectively. It is noteworthy that the events of the slow and fast states are triggered at different time and different system has different triggering instants. Table I shows that the cost and triggering numbers are different with different $\beta, \delta$ and it would require more cost with fewer triggering numbers.

TABLE I

THE COST AND TRIGGERING NUMBERS

\begin{tabular}{|c|c|c|c|c|c|c|c|}
\hline & & \multicolumn{6}{|c|}{ Triggering numbers (T=15s) } \\
\cline { 3 - 8 } Parameters & Cost & $n_{1 s}$ & $n_{1 f}$ & $n_{2 s}$ & $n_{2 f}$ & $n_{3 s}$ & $n_{3 f}$ \\
\hline $\begin{array}{c}\beta=3.3 \times 10^{-5} \\
\delta=2 e^{-0.5 t}\end{array}$ & 1.3121 & 43 & 42 & 63 & 64 & 22 & 32 \\
\hline $\begin{array}{c}\beta=0 \\
\delta=2 e^{-0.5 t}\end{array}$ & 1.3153 & 44 & 43 & 64 & 61 & 22 & 32 \\
\hline $\begin{array}{c}\beta=3.3 \times 10^{-5} \\
\delta=e^{-0.6 t}\end{array}$ & 1.2351 & 181 & 158 & 212 & 202 & 81 & 94 \\
\hline $\begin{array}{c}\beta=0 \\
\delta=e^{-0.6 t}\end{array}$ & 1.2354 & 225 & 179 & 308 & 255 & 87 & 97 \\
\hline
\end{tabular}

When the uncertain interconnected TTSSs (1) are considered, where $A, B$ have the same definition in above and for
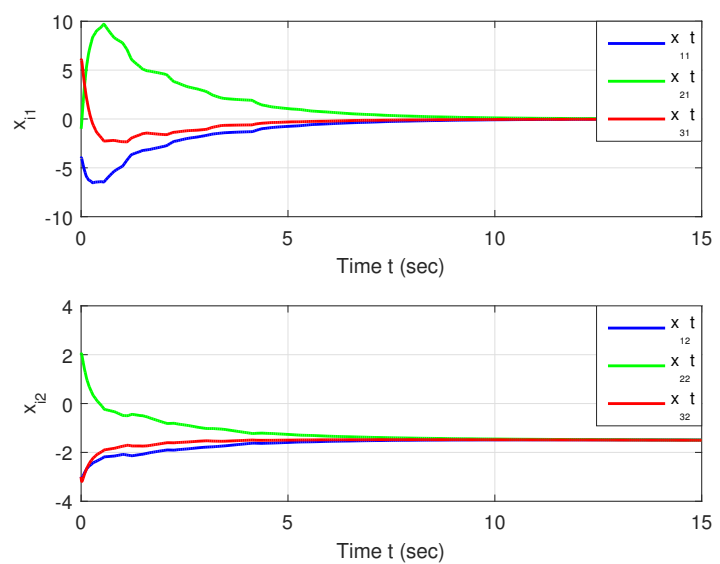

(a) The slow state trajectories of each system.
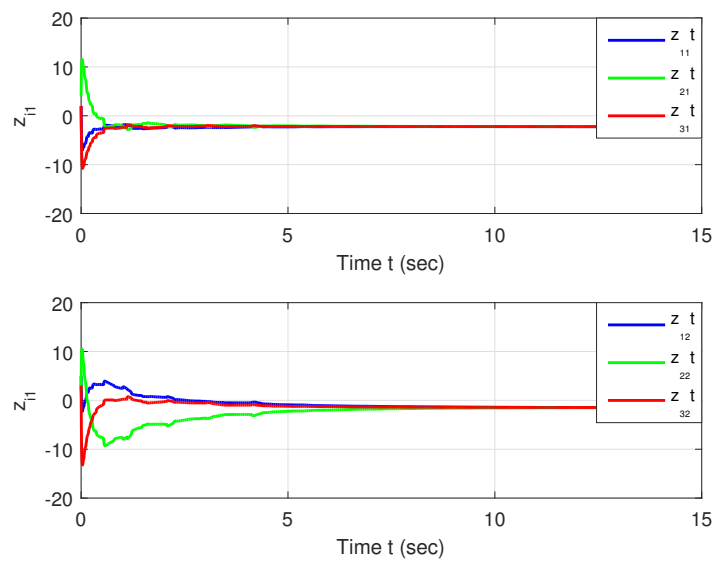

(b) The fast state trajectories of each system.

Fig. 2. The simulation results of Theorem 3 .
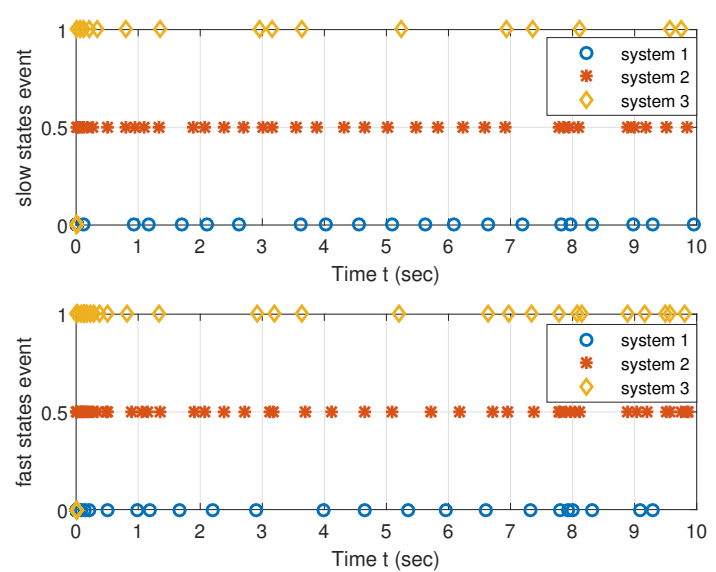

Fig. 3. The event-triggering time of each system.

$i=1,2,3$,

$$
\Xi_{i}=\left(\begin{array}{cccc}
0.01 i & 0.02 i & 0 & 0 \\
0 & -0.01 i & 0.01 i & 0 \\
0 & 0 & 0.02 i & 0.01 i \\
0.01 i & 0 & 0 & 0
\end{array}\right)
$$


Choosing same $Q_{1}, Q_{2}$ as above, then same $K_{1}, K_{2}$ can be obtained. Then it can be obtained that $\beta=1.7 \times 10^{-5}$. Let $\beta_{i j}=\beta, \delta_{i j}=2 e^{-0.5 t}$, for $i=1,2, j=1,2,3$. Then, the simulation result of Theorem 3 is shown in Fig. 2-3. Fig. 2 shows that consensus of both the slow and fast states are achieved at a cost of $J=1.237$ and confirms the effectiveness of Theorem 3. Fig. 3 plots the triggering instants determined by the designed event-triggered mechanisms.

Example 2: Let us now consider three interconnected DC motors. Since the dynamic described by the electromagnetic equilibrium equation is much faster than the one described by torque equation, interconnected DC-motors can be modeled as interconnected TTSSs. As in [37], the dynamics of the nominal interconnected DC-motors are as follows

$$
\begin{aligned}
& J_{m} \frac{d \omega_{i}}{d t}=-b \omega_{i}+k_{m} I_{i}, \\
& \bar{L} \frac{d I_{i}}{d t}=-k_{b} \omega_{i}-R_{0} I_{i}+u_{i} .
\end{aligned}
$$

where $i=1,2,3, I_{i}, u_{i}, \omega_{i}$ denote the armature current, voltage, and angular speed, $R_{0}=0.6 \Omega$ is the resistance, $J_{m}=0.093 \mathrm{~kg} \cdot \mathrm{m}^{2}$ is the equivalent moment of inertia, $b=0.008$ is the equivalent viscous friction coefficient while $k_{m}=0.7274 \mathrm{~N} \cdot \mathrm{m}, k_{b}=560.6 \mathrm{v} \cdot \mathrm{s} / \mathrm{rad}$ are respectively the torque and back e.m.f. developed with constant excitation flux. Finally $L=0.006 H$ is the inductance which is very small and plays the role of the singular perturbation parameter of the system. Thus, system (36) can be described by system (1) with $A_{11}=-0.086, A_{12}=7.82, A_{11}=-0.6, A_{12}=0.6$, $\varepsilon=0.006$. Here, we consider the structured uncertainties $\Xi_{i}=\left(\begin{array}{cc}0.01 i & 0 \\ 2 i & i\end{array}\right), i=1,2,3$.
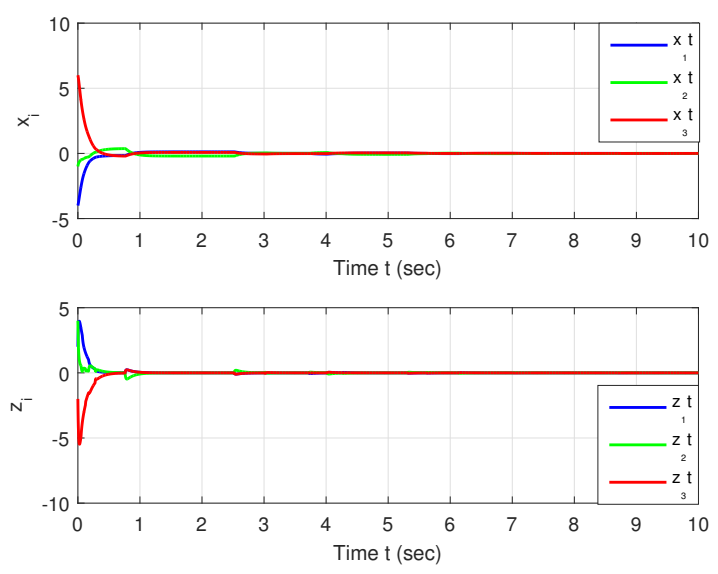

Fig. 4. The simulation results of Theorem 2 .

The initial condition of system is taken as $\left(x_{1}(0), z_{1}(0)\right)=$ $(-4,2),\left(x_{2}(0), z_{2}(0),\right)=(-1,4),\left(x_{3}(0), z_{3}(0)\right)=(6,-2)$. The global cost $J$ is also defined as in 34. The interaction topology $\mathcal{G}$ is also given in Figure 1. Thus, Assumption 1 holds. Choose $g_{m}=g_{M}=1, Q_{1}=Q_{2}=10 I_{2}$. Then, it can be obtained that $P_{1}=0.0063, P_{2}=0.083, K_{1}=0.1218$, $K_{2}=0.0830$. Similarly, Let $\beta_{i j}=0, \delta_{i j}=2 e^{-0.5 t}$, for $i=1,2, j=1,2,3$. Then, simulation results of Theorem 3 are shown in Fig.4-5. Fig. 4 shows that consensus of both the
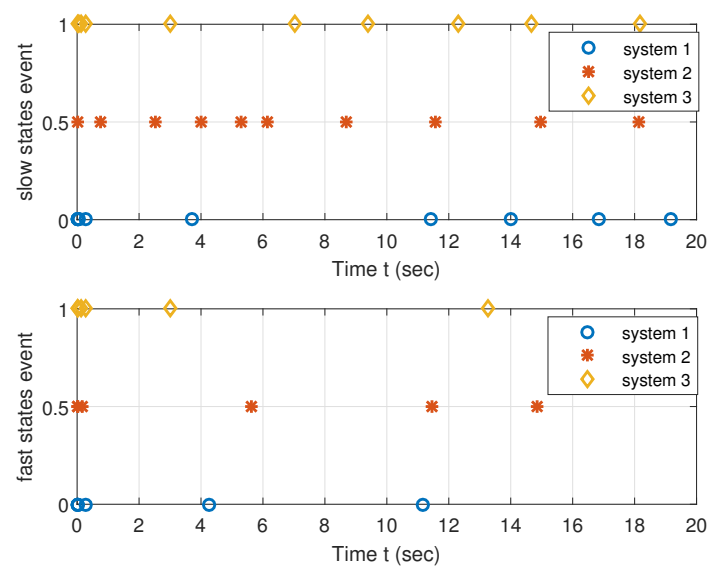

Fig. 5. The event-triggering time of each system.

slow and fast states are achieved at a cost of $J=0.0083$ and confirms the effectiveness of Theorem 3. Fig. 5 plots the triggering instants determined by the designed event-triggered mechanisms.

\section{CONCLUSION}

In this paper, we have investigated event-triggered consensus problem with guaranteed cost for a class of interconnected TTSSs. Due to the two-time-scale property of each system, two event-triggered mechanisms are designed to independently decide the sampling and transmitting instants for the slow and fast states respectively. Besides, by using the Chang transformation, an event-triggered controller is designed and combined with the two proposed event-triggered mechanisms to achieve guaranteed-cost consensus for the interconnected nominal TTSSs with fixed interaction topology. Zeno behavior is also excluded for each system to ensure the practical implementation of the proposed event-triggered strategies. Furthermore, the results take into account structural uncertainties on the interconnected TTSSs. Basically our results hold as far as the uncertainties satisfy a norm condition. Further considerations include the consensus of interconnected TTSSs with time-delay or with nonlinear dynamics via event-triggered strategies.

\section{REFERENCES}

[1] F. Bullo, J. Cortes, and S. Martinez, Distributed control of robotic networks: a mathematical approach to motion coordination algorithms. Princeton University Press, 2009, vol. 27.

[2] Y.-W. Wang, Y.-W. Wei, X.-K. Liu, N. Zhou, and C. G. Cassandras, "Optimal persistent monitoring using second-order agents with physical constraints," IEEE Transactions on Automatic Control, vol. 64, no. 8, pp. 3239-3252, 2018.

[3] E. Steur, I. Tyukin, and H. Nijmeijer, "Semi-passivity and synchronization of diffusively coupled neuronal oscillators," Physica D: Nonlinear Phenomena, vol. 238, no. 21, pp. 2119-2128, 2009.

[4] W. Michiels, C.-I. Morărescu, and S.-I. Niculescu, "Consensus problems with distributed delays, with application to traffic flow models," SIAM Journal on Control and Optimization, vol. 48, no. 1, pp. 77-101, 2009.

[5] I.-C. Morarescu and A. Girard, "Opinion dynamics with decaying confidence: Application to community detection in graphs," IEEE Transactions on Automatic Control, vol. 56, no. 8, pp. 1862-1873, 2010. 
[6] T. Ma, B. Cui, Y. Wang, and K. Liu, "Stochastic synchronization of delayed multiagent networks with intermittent communications: An impulsive framework," International Journal of Robust and Nonlinear Control, vol. 29, no. 13, pp. 4537-4561, 2019.

[7] Y. Wu, H. Su, P. Shi, Z. Shu, and Z.-G. Wu, "Consensus of multiagent systems using aperiodic sampled-data control," IEEE Transactions on Cybernetics, vol. 46, no. 9, pp. 2132-2143, 2015.

[8] Y.-W. Wang, X.-K. Liu, J.-W. Xiao, and X. Lin, "Output formationcontainment of coupled heterogeneous linear systems under intermittent communication," Journal of the Franklin Institute, vol. 354, no. 1, pp. 392-414, 2017.

[9] Y. Fan, G. Feng, Y. Wang, and C. Song, "Distributed event-triggered control of multi-agent systems with combinational measurements," $\mathrm{Au}$ tomatica, vol. 49, no. 2, pp. 671-675, 2013.

[10] X. Ge, Q. Han, L. Ding, Y. Wang, and X. Zhang, "Dynamic eventtriggered distributed coordination control and its applications: A survey of trends and techniques," IEEE Transactions on Systems, Man, and Cybernetics: Systems, vol. 50, no. 9, pp. 3112-3125, 2020.

[11] L. Ding, Q. Han, and X. Zhang, "Distributed secondary control for active power sharing and frequency regulation in islanded microgrids using an event-triggered communication mechanism," IEEE Transactions on Industrial Informatics, vol. 15, no. 7, pp. 3910-3922, 2019.

[12] X.-M. Zhang, Q.-L. Han, and B.-L. Zhang, "An overview and deep investigation on sampled-data-based event-triggered control and filtering for networked systems," IEEE Transactions on Industrial Informatics, vol. 13, no. 1, pp. 4-16, 2016.

[13] D. V. Dimarogonas, E. Frazzoli, and K. H. Johansson, "Distributed event-triggered control for multi-agent systems," IEEE Transactions on Automatic Control, vol. 57, no. 5, pp. 1291-1297, 2012.

[14] E. Garcia, Y. Cao, H. Yu, P. Antsaklis, and D. Casbeer, "Decentralised event-triggered cooperative control with limited communication," International Journal of Control, vol. 86, no. 9, pp. 1479-1488, 2013.

[15] G. S. Seyboth, D. V. Dimarogonas, and K. H. Johansson, "Event-based broadcasting for multi-agent average consensus," Automatica, vol. 49 , no. 1 , pp. 245-252, 2013

[16] O. Demir and J. Lunze, "Event-based synchronisation of multi-agent systems," IFAC Proceedings Volumes, vol. 45, no. 9, pp. 1-6, 2012

[17] D. Yang, W. Ren, X. Liu, and W. Chen, "Decentralized event-triggered consensus for linear multi-agent systems under general directed graphs," Automatica, vol. 69, no. C, pp. 242-249, 2016.

[18] W. Hu, L. Liu, and G. Feng, "Consensus of linear multi-agent systems by distributed event-triggered strategy," IEEE Transactions on Cybernetics, vol. 46, no. 1, pp. 148-157, 2016.

[19] D. Ye, M. M. Chen, and H. J. Yang, "Distributed adaptive eventtriggered fault-tolerant consensus of multiagent systems with general linear dynamics," IEEE Transactions on Cybernetics, vol. 49, no. 3, pp. 757-767, 2019.

[20] W. Hu, L. Liu, and G. Feng, "Output consensus of heterogeneous linear multi-agent systems by distributed event-triggered/self-triggered strategy," IEEE Transactions on Cybernetics, vol. 47, no. 8, pp. 19141924, 2017.

[21] R. Wang, T. Zhou, Z. Jing, and L. Chen, "Modelling periodic oscillation of biological systems with multiple timescale networks," Systems Biology, vol. 1, no. 1, pp. 71-84, 2004.

[22] A. Zagaris, H. G. Kaper, and T. J. Kaper, "Analysis of the computational singular perturbation reduction method for chemical kinetics," Journal of Nonlinear Science, vol. 14, no. 1, pp. 59-91, 2004

[23] S. Ball, E. Barany, S. Schaffer, and K. Wedeward, "Nonlinear controllability of singularly perturbed models of power flow networks," International Journal of Pure and Applied Mathematics, vol. 47, no. 2, pp. 243-266, 2008.

[24] N. Jiang and H.-D. Chiang, "A two-time scale dynamic correction method for fifth-order generator model undergoing large disturbances," IEEE Transactions on Power Systems, vol. 31, no. 5, pp. 3616-3623, 2015.

[25] H. Yoo and Z. Gajic, "New designs of linear observers and observerbased controllers for singularly perturbed linear systems," IEEE Transactions on Automatic Control, vol. 63, no. 11, pp. 3904-3911, 2018.

[26] H. Jardón-Kojakhmetov, J. M. Scherpen, and D. del Puerto-Flores, "Stabilization of a class of slow-fast control systems at non-hyperbolic points," Automatica, vol. 99, pp. 13-21, 2019.

[27] J. Xu, Y. Niu, E. Fridman, and L. Fridman, "Finite frequency h control of singularly perturbed euler-lagrange systems: An artificial delay approach," International Journal of Robust and Nonlinear Control, vol. 29 , no. 2 , pp. 353-374, 2019.
[28] J. Song and Y. Niu, "Dynamic event-triggered sliding mode control: Dealing with slow sampling singularly perturbed systems," IEEE Transactions on Circuits and Systems II: Express Briefs, vol. 67, no. 6, pp. 1079-1083, 2020.

[29] W. Yang, Y.-W. Wang, J.-W. Xiao, and W.-H. Chen, "Modulus consensus in a network of singularly perturbed systems with collaborative and antagonistic interactions," International Journal of Control, vol. 90 , no. 12 , pp. 2667-2676, 2017.

[30] W. Yang, Y.-W. Wang, J.-W. Xiao, and Z.-W. Liu, "Coordination of networked delayed singularly perturbed systems with antagonistic interactions and switching topologies," Nonlinear Dynamics, vol. 89, no. 1, pp. 741-754, 2017.

[31] J. B. Rejeb, I.-C. Morărescu, and J. Daafouz, "Control design with guaranteed cost for synchronization in networks of linear singularly perturbed systems," Automatica, vol. 91, pp. 89-97, 2018.

[32] K. Sivaranjani, R. Rakkiyappan, J. Cao, and A. Alsaedi, "Synchronization of nonlinear singularly perturbed complex networks with uncertain inner coupling via event triggered control," Applied Mathematics and Computation, vol. 311, pp. 283-299, 2017.

[33] H. Jaleel and J. S. Shamma, "Decentralized energy aware cooptimization of mobility and communication in multiagent systems," in 2016 IEEE 55th Conference on Decision and Control (CDC). IEEE, 2016, pp. 2665-2670.

[34] L. Xing, C. Wen, Z. Liu, H. Su, and J. Cai, "Event-triggered adaptive control for a class of uncertain nonlinear systems," IEEE Transactions on Automatic Control, vol. 62, no. 4, pp. 2071-2076, 2017.

[35] M. Bhandari, D. M. Fulwani, and R. Gupta, "Event-triggered composite control of a two time scale system," IEEE Transactions on Circuits and Systems II: Express Briefs, vol. 65, no. 4, pp. 471-475, 2017.

[36] Z. Bien and J.-H. Kim, "A robust stability bound of linear systems with structured uncertainty," IEEE Transactions on Automatic Control, vol. 37, no. 10, pp. 1549-1551, 1992.

[37] C. Yang, S. Zhong, X. Liu, W. Dai, and L. Zhou, "Adaptive composite suboptimal control for linear singularly perturbed systems with unknown slow dynamics," International Journal of Robust and Nonlinear Control, vol. 30 , no. 7 , pp. $2625-2643,2020$

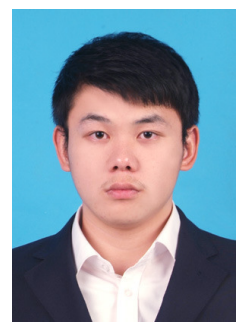

Yan Lei received the B.S. degree from Hohai University, Changzhou, China, in 2016. He is currently pursuing the Ph.D. degree in control theory and control engineering with the Huazhong University of Science and Technology, Wuhan, China. His research interests include hybrid systems, multi-agent systems, and event-triggered control

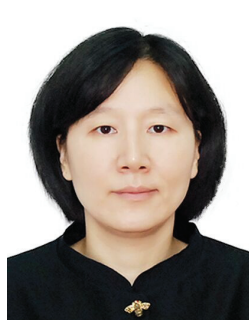

Yan-Wu Wang (M10-SM13)received the B.S. degree in automatic control, the M.S. degree and the $\mathrm{Ph} . \mathrm{D}$. degree in control theory and control engineering from Huazhong University of Science and Technology (HUST), Wuhan, China, in 1997, 2000, and 2003, respectively. She has been a Professor with the School of Artificial Intelligence and Automation, HUST, since 2009. She is also with the Key Laboratory of Image Processing and Intelligent Control, Ministry of Education, China. Her research interests include hybrid systems, cooperative control, and multi-agent systems with applications in smart grid.

Dr. Wang was a recipient of several awards, including the first prize of Hubei Province Natural Science in 2014, the first prize of the Ministry of Education of China in 2005, and the Excellent PhD Dissertation of Hubei Province in 2004, China. In 2008, she was awarded the title of "New Century Excellent Talents" by the Chinese Ministry of Education. 


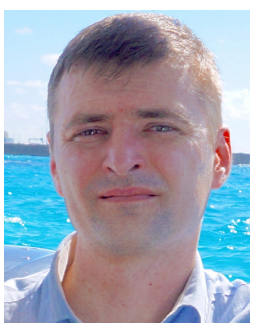

Irinel Constantin Morărescu is currently Full Professor at Universit de Lorraine and researcher at the Research Centre of Automatic Control (CRAN UMR 7039 CNRS) in Nancy, France. He received the B.S. and the M.S. degrees in Mathematics from University of Bucharest, Romania, in 1997 and 1999, respectively. In 2006 he received the Ph.D. degree in Mathematics and in Technology of Information and Systems from University of Bucharest and University of Technology of Compigne, respectively. He received the "Habilitation Diriger des Recherches" from the Universit de Lorraine in 2016. His works concern stability and control of time-delay systems, stability and tracking for different classes of hybrid systems, consensus and synchronization problems.

Irinel-Constantin Morărescu is on the editorial board of Nonlinear Analysis:Hybrid Systems and of IEEE Control Systems Letters. He is a member of the IEEE Control Systems Society- Conference Editorial Board and member of the IFAC Technical Committee on Networked Systems.

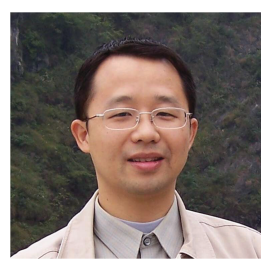

Jiang-Wen Xiao received the B.S. degree in electrical engineering, the M.S. degree in control theory and control engineering, and the Ph.D. degree in systems engineering from Huazhong University of Science and Technology (HUST), Wuhan, China, in 1994, 1997, and 2001 respectively. From 2001 to 2003, he worked as a Post-Doctoral Researcher in the College of Management, HUST, Wuhan, China. He is currently a Professor in the School of Artificial Intelligence and Automation, HUST. His research interests include modeling and control of nonlinear and complex systems, data mining, and optimization. 